\section{Effect of Bisphenol A on the Feeding Behavior of Caenorhabditis elegans}

\author{
Shinya Kohra, ${ }^{*, a}$ Kazuko Kuwahara, ${ }^{a}$ \\ Yuji Takao, ${ }^{a}$ Yasuhiro Ishibashi, ${ }^{b}$ \\ Ho Chul Lee, ${ }^{c}$ Koji Arizono, ${ }^{d}$ \\ and Nobuaki Tominaga ${ }^{e}$
}

${ }^{a}$ Faculty of Environmental Sciences, ${ }^{b}$ Environmental Protection Center, and 'Graduate School of Science and Technology, Nagasaki University, 1-14 Bunkyo-machi, Nagasaki 852-8521, Japan, ${ }^{d}$ Faculty of Environmental and Symbiotic Sciences, Prefectural University of Kumamoto, 3-1-100 Tsukide, Kumamoto 862-8502, Japan, and ${ }^{~}$ Department of Chemical and Biological Engineering, Ariake National College of Technology, 150 Higashihagino-machi, Omuta 836-8585, Japan

(Received September 26, 2001; Accepted October 10, 2001)

We observed and evaluated the feeding behavior of the free-living nematode Caenorhabditis elegans $(C$. elegans) after exposure to bisphenol A (BPA) and nonylphenol (NP). Exposed organisms were transferred to chemical-free culture medium and their attainment levels (the number of worms reaching the food source divided by the total number of worms on the Petri plate) were recorded after $2,4,6,8$, and $24 \mathrm{hr}$. Results showed a significant decrease in the attainment level of worms exposed to $10 \mu \mathrm{M}$ and $0.1 \mu \mathrm{M}$ BPA. However, there was a slight increase in the attainment level of nematodes treated with $1 \mu \mathrm{M}$ NP. These results differ from previous studies showing NP as being more lethal to nematodes than BPA.

Key words — feeding behavior, Caenorhabditis elegans, bisphenol A, nonylphenol, biosensor

\section{INTRODUCTION}

Experiments have been carried out in order to evaluate the toxicity of chemical substances using various animals and toxicological endpoints. $C$. elegans is a free-living nematode with a completely mapped genome that has become a commonly used laboratory model organism for molecular biology, cellular biology, etc. ${ }^{1,2)}$ Recently, C. elegans has also been used for the toxicity studies of the chemical

\footnotetext{
*To whom correspondence should be addressed: Faculty of Environmental Sciences, Nagasaki University, 1-14 Bunkyo-machi, Nagasaki 852-8521, Japan. Tel.: +81-95-843-6379; Fax: +8195-843-6377; E-mail: kohra@net.nagasaki-u.ac.jp
}

substances and environmental samples. ${ }^{3-8)}$ Biosensors using $C$. elegans represent a more complex level of biological organization and a higher trophic level compared to bacterial, yeast and cell culture biosensors. ${ }^{9-11)} C$. elegans is also easily grown in the laboratory compared to other organisms such as fish and mice, ${ }^{12,13)}$ which are often used in in vivo assays.

Toxicant evaluation has several endpoints, with lethality and reproduction often used. ${ }^{3)}$ Recently, we utilized $C$. elegans in the toxicity evaluation of chemical substances..$^{14,15}$ In this study, we examined the feeding behavior of the nematode as a biological parameter after exposure to bisphenol A (BPA) or nonylphenol (NP).

\section{MATERIALS AND METHODS}

Wild type C.elegans were grown and maintained as described by Brenner (1974), and routinely cultured at $20^{\circ} \mathrm{C}$ on glass, acid-washed petri plates containing nematode growing media (NGM) agar and Escherichia coli (E. coli) as food source. ${ }^{1)}$

The ages of the nematodes were initially determined. Two and half-day old worms were appropriate because they moved smoothly to the food source unlike the younger and older ones. Adult worms often do not migrate when ovulation begins.

The testing procedure is as follows. Eggs were placed on E. coli-free NGM agar plates and incubated for $15-20 \mathrm{hr}$ at $20^{\circ} \mathrm{C}$. Juvenile worms (first stage) were then transferred to each NGM agar plate containing the chemicals and a lawn of E. coli. The experimental plates consisted of two concentrations $(10 \mu \mathrm{M}$ and $0.1 \mu \mathrm{M})$ of BPA, two concentrations $(1 \mu \mathrm{M}$ and $0.1 \mu \mathrm{M})$ of NP, and two controls, one containing only medium and the other containing dimethyl sulfoxide (DMSO) $(5.0 \mathrm{~g} / \mathrm{l})$, which was used as the solvent for BPA and NP. The set-ups were then incubated at $20^{\circ} \mathrm{C}$ for $46 \mathrm{hr}$, and then the worms transferred to chemical-free $9 \mathrm{~cm}$ plates for the feeding behavior assay. On the latter, E. coli was grown circularly within a $0.5 \mathrm{~cm}$ radius from the center. The transfer of the exposed worms was carried out as follows. Each exposed worm was rinsed with M9 buffer in 15-ml glass centrifuge tubes and centrifuged at $800 \mathrm{rpm}$ for $30 \mathrm{sec}$. After the supernatants were removed, the pelleted worms were rinsed thrice with M9 buffer as described earlier to eliminate bacteria. The washed worms were then resuspended in M9 buffer and then transferred one by one with $2 \mu \mathrm{l}$ 


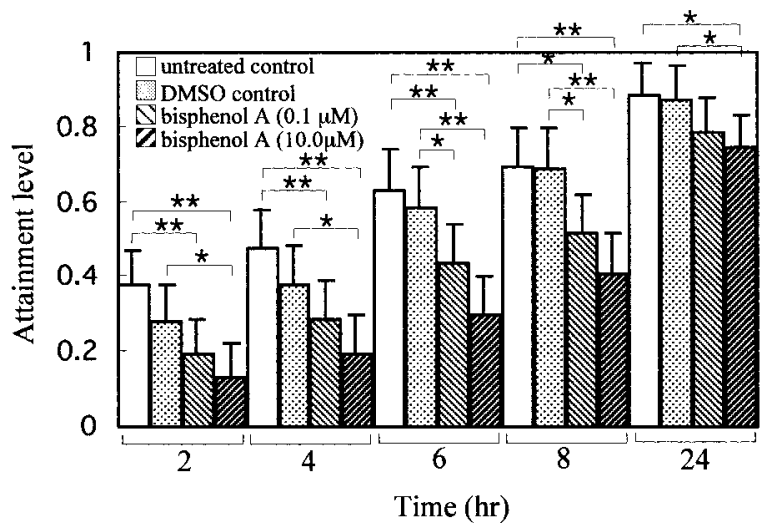

Fig. 1. Influence of BPA in Attainment Level of C. elegans SEM. ${ }^{*} p<0.05,{ }^{* *} p<0.01$ statistically significant. Error bar represents

M9 onto the NGM agar. The worms were evenly placed $4 \mathrm{~cm}$ from the center. Each test plate was loaded with 12 worms and five replicates were set up for each treatment ( 60 worms total per treatment). The testing was replicated five times (300 worms total per treatment). After 2, 4, 6, 8, and $24 \mathrm{hr}$ of incubation at $20^{\circ} \mathrm{C}$, the number of nematodes that have reached the $E$. coli colony was counted in each plate using a dissecting microscope. The attainment level of C. elegans was obtained by dividing the number of worms that reached the food source by the total number of worms on the plate.

\section{RESULTS AND DISCUSSION}

All worms survived during the experiment. As shown in Fig. 1, there was no evidence $(p>0.05)$ of altered attainment levels between the untreated control and the DMSO control. However, when the $C$. elegans were exposed to $10 \mu \mathrm{M} \mathrm{BPA}$, the attainment levels decreased significantly $(p<0.05)$ compared to the untreated control in all observation points. The decrease was similarly significant $(p<0.05)$ using $0.1 \mu \mathrm{M} \mathrm{BPA}$ at $2,4,6$, and $8 \mathrm{hr}$. At the higher concentration, the difference was more significant $(p<0.01)$ between 2 to $8 \mathrm{hr}$. However, a comparison between the attainment levels of the organism treated with $0.1 \mu \mathrm{M}$ and $10 \mu \mathrm{M}$ BPA showed no significant difference. Results using NP as shown in Fig. 2 do not reveal any significant differences between treatments and controls at 2, 4, 6, and $8 \mathrm{hr}$. A $24 \mathrm{hr}$ exposure to $1 \mu \mathrm{M}$ NP led to an increase $(p<0.05)$ in the attainment level of $C$. elegans. Considering that NP is generally more lethal to $C$. elegans than BPA, these results are interesting. After expo-

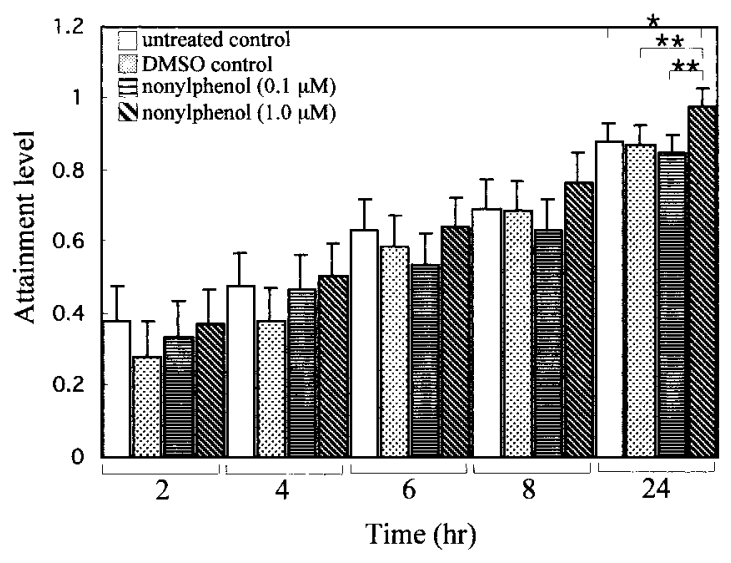

Fig. 2. Influence of NP in Attainment Level of C. elegans SEM.

${ }^{*} p<0.05,{ }^{* *} p<0.01$ statistically significant. Error bar represents

sure to these compounds, the activity of the worms appeared no different from the controls as observed under a dissecting microscope. There were no significant differences either in their respiration volumes when the oxygen consumption of approximately 200 worms in M9 buffer was measured using a disposable oxygen electrode sensor and multichannel dissolved oxygen meters (DOX-96). ${ }^{16)}$ In this study, we indicated that $C$. elegans have a good responsibility for BAP using the feeding behavior as a biological parameter. Thus it seems that this parameter will be able to become interesting test endpoint for chemical hazard assessment.

Acknowledgements The authors are grateful to DAIKIN INDUSTRIES, Ltd. (Osaka) for providing disposable oxygen electrode sensors and multichannel dissolved oxygen meters (DOX-96). This work was supported by Japan Society for the Promotion of Science (Grant-in Aid for Scientific Research (C) 13833009).

\section{REFERENCES}

1) Brenner, S. J. (1974) The genetics of Caenorhabditis elegans. Genetics, 77, 71-94.

2) Sulston, J. and Hodgkin, J. (1988) Methods. In The nematode Caenorhabditis elegans, (Wood, W. B. and the community of $C$. elegans Researchers, Eds.), Cold Spring Harbor, NY: Cold Spring Harbor Laboratory, N.Y., pp. 587-606.

3) Dhawan, R., Dusenbery, D. B. and Williams, P. L. (1999) Comparison of lethality, reproduction, and behavior as toxicological endpoints in the nematode Caenorhabditis elegans. J. Toxicol. Environ. Health 
A, 58, 451-462.

4) Hoss, S., Haitzer, M., Traunspurger, W. and Steinberg, C. E. W. (1999) Growth and fertility of Caenorhabditis elegans (nematoda) in unpolluted freshwater sediments: response to particle size distribution and organic content. Environ. Toxicol. Chem., 18, 2921-2925.

5) Peradney, C. L. and Williams, P. L. (2000) Utility of Caenorhabditis elegans for assessing heavy metal contamination in artificial soil. Arch. Environ. Contam. Toxicol., 39, 113-118.

6) Hood, T. E., Calabrese, E. J. and Zuckerman, B. M. (2000) Detection of an estrogen receptor in two nematode species and inhibition of binding and development by environmental chemicals. Ecotoxicol. Environ. Saf., 47, 74-81.

7) Williams, P. L., Anderson, G. L., Johnstone, J. L., Nunn, A. D., Tweedle, M. F. and Wedeking, P. (2000) Caenorhabditis elegans as an alternative animal species. J. Toxicol. Environ. Health A, 61, 641-647.

8) Lagido, C., Pettitt, J., Porter, A. J. R., Paton, G. I. and Glover, L. A. (2001) Development and application of bioluminescent Caenorhabditis elegans as multicellular eukaryotic biosensors. FEBS Lett., 493, 36-39.

9) Hollis, R. P., Killham, K. and Glover, L. A. (2000) Design and application of a biosensor for monitoring toxicity of compounds to eukaryotes. Appl. Environ. Microbiol, 66, 1676-1679.

10) Nishihara, T., Nishikawa, J., Kanayama, T., Dakeyama, F., Saito, K., Imagawa, M., Takatori, S., Kitagawa, Y., Hori, S. and Utsumi, H. (2000) Estrogenic activities of 517 chemicals by yeast two- hybrid assay. J. Health Sci., 46, 282-298.

11) Makela, S., Davis, V., Tally, W. C., Korkman, H., Salo, L., Santti, R., Vihko, R. and Korach, K. S. (1994) Dietary estrogens act through receptor mediated processes and show no antiestrogenicity in cultured breast cancer cells. Environ. Health Perspect., 102, 572-578.

12) Shioda, T. and Wakabayashi, M. (2000) Effect of certain chemicals on the reproduction of medaka (Oryzias latipes). Chemosphere, 40, 239-243.

13) vom Saal, F. S., Cooke, P. S., Buchanan, D. L., Palanza, P., Thayer, K. A., Nagel, S. C., Parmigiani, S. and Welshons, W. V. (1998) A physiologically based approach to the study of bisphenol A and other estrogenic chemicals on the size of reproductive organs, daily sperm production, and behavior. Toxicol. Ind. Health, 14, 239-260.

14) Kohra, S., Tominaga, N., Mitsui, Y., Takao, Y., Ishibashi, Y. and Arizono, K. (1999) Determination of a screening system of endocrine disruptors by the induction of Vitellogenin mRNA in C. elegans larvae. J. Health Sci., 45, P-37.

15) Miwa, T., Shimada, H., Tominaga, N., Mitsui, Y., Kohra, S. and Arizono, K. (1999) The establishment of the new safety test with C. elegans - the case of trace elements - Biomed. Res. Trace Elements, 10, 285-286.

16) Amano, Y., Okumura, C., Yoshida, M., Katayama, H., Unten, S., Arai, J., Tagawa, T., Hoshino, S., Hashimoto, H. and Ishikawa, H. (1999) Measuring respiration of cultured cell with oxygen electrode as a metabolic indicator for drug screening. Hum. Cell, 112, 3-10. 